\title{
Perfil epidemiológico da doença de Chagas aguda na região norte do Brasil no ano de 2015-2019
}

\author{
Epidemiological profile of acute Chagas disease in northern Brazil in 2015-2019
}

\begin{abstract}
Perfil epidemiológico de la enfermedad de Chagas aguda en el norte de Brasil en 2015-2019
\end{abstract}
Sabrinna Barbosa de Souza ${ }^{1 *}$, Andressa de Oliveira ${ }^{1}$, Ester de Sousa Campos ${ }^{1}$, Gabriela Assunção Godinho1, Ana Flávia Fernandes Saraiva1', Beatriz Moura Araujo¹, Andressa Batista Veras de Menezes $^{2}$, Layanne Araújo Silva ${ }^{1}$, Renata de Souza Meireles¹, Elyanne dos Santos Gomes².

\section{RESUMO}

Objetivo: Traçar o perfil epidemiológico através das variáveis sexo, faixa etária, raça/cor, zona de residência, local provável de infecção e capital de notificação da Doença de Chagas Aguda (DCA) na região norte do Brasil no ano de 2015-2019. Métodos: Trata-se de um estudo epidemiológico, retrospectivo, exploratório e descritivo, realizado através dos dados secundários do Sistema de Informação de Agravos de Notificação (SINAN) disponibilizados no sitio eletrônico do Departamento de Informática do Sistema Único de Saúde (DATASUS), onde foram estudadas as notificações de Doença de Chagas Aguda na região Norte, no período de 2015 a 2019. Resultados: A região Norte obteve a maior incidência de casos notificados no Brasil com 8,84/100.000 ha, porém no ano de 2018 houve maior notificação de DCA com incidência de 1,89/100.000 ha. Revelou-se predominância nos indivíduos masculinos (54,29\%), raça/cor parda (84,78\%) e indivíduos de 20 39 anos $(34,44 \%)$ na zona rural $(51,39 \%)$. Conclusão: Possibilitou identificar o perfil epidemiológico e a distribuição espacial da doença, fatores importantes para gerar informações para a vigilância de saúde, criação de medidas intervencionistas para prevenção da doença.

Palavras-chave: Doença de Chagas, Epidemiologia, Trypanosoma cruzi.

\begin{abstract}
Objective: To trace the epidemiological profile through the variables sex, age group, race / color, area of residence, probable place of infection and notification capital of Acute Chagas Disease (DCA) in the northern region of Brazil in 2015-2019. Methods: This is an epidemiological, retrospective, exploratory and descriptive study, carried out using secondary data from the Notifiable Diseases Information System (SINAN) available on the website of the Department of Informatics of the Unified Health System (DATASUS), where they were Acute Chagas Disease notifications in the North region were studied in the period from 2015 to 2019. Results: The North region had the highest incidence of cases reported in Brazil with 8.84 / 100,000 ha, however in the year 2018 there was a higher notification of DCA with an incidence of 1.89 / 100,000 ha. There was a predominance of male individuals $(54.29 \%)$, race / brown color $(84.78 \%)$ and individuals aged 20-39 years $(34.44 \%)$ in rural areas $(51.39 \%)$. Conclusion: It made it possible to identify the epidemiological profile and the spatial distribution of the disease, important factors to generate information for health surveillance, creation of interventionist measures to prevent the disease.
\end{abstract}

Keywords: Chagas disease, Epidemiology, Trypanosoma cruzi.

\footnotetext{
${ }^{1}$ Instituto Tocantinense Presidente Antônio Carlos (ITPAC), Porto Nacional - TO.

*E-mail: sabrinnasbds@gmail.com

${ }^{2}$ Universidade de Rio Verde (UNIRV), Goianésia - GO.
}

SUBMETIDO EM: 6/2021

ACEITO EM: 6/2021

PUBLICADO EM: 7/2021 


\section{RESUMEN}

Objetivo: Trazar el perfil epidemiológico a través de las variables sexo, grupo de edad, raza / color, área de residencia, lugar probable de infección y capital de notificación de la Enfermedad de Chagas Aguda (DCA) en la región norte de Brasil en el año 2015 -2019. Métodos: Se trata de un estudio epidemiológico, retrospectivo, exploratorio y descriptivo, realizado a partir de datos secundarios del Sistema de Información de Enfermedades Notificables (SINAN) disponibles en el sitio web del Departamento de Informática del Sistema Único de Salud (DATASUS), donde se encontraban Chagas Agudo Las notificaciones de enfermedades en la región Norte se estudiaron en el período de 2015 a 2019. Resultados: La región Norte tuvo la mayor incidencia de casos reportados en Brasil con 8.84 / 100,000 ha, sin embargo, en el año 2018 hubo una mayor notificación de DCA con una incidencia de 1,89 / 100.000 ha. Predominó el sexo masculino (54,29\%), la raza / color marrón (84,78\%) y los individuos de 20 a 39 años (34,44\%) en las zonas rurales (51,39\%). Conclusión: permitió identificar el perfil epidemiológico y la distribución espacial de la enfermedad, factores importantes para generar información para la vigilancia en salud, creación de medidas intervencionistas para prevenir la enfermedad.

Palabras clave: Enfermedad de Chagas, Epidemiología, Trypanosoma cruzi.

\section{INTRODUÇÃO}

A descoberta da Doença de Chagas (DC) ocorreu no ano de 1909 pelo pesquisador do Instituto Oswaldo Cruz chamado Carlos Ribeiro Justiniano das Chagas, no qual com o intuito de combater a malária no interior de Minas Gerais acabou encontrando um protozoário flagelado que o denominou de Trypanosoma minasense. Ao fazer mais pesquisas percebeu que nos insetos triatomíneos existiam outro tipo de Trypanosoma chamando-o de Trypanosoma cruzi, o responsável pela DC (CARVALHO GLB, et al., 2018).

O Trypanosoma cruzi pertencente à família Trypanosomatidae, possui pleomorfismo, possui estrutura e biologia diferenciadas que se evidencia no seu ciclo de vida heteroxênico. O parasita apresenta-se de variadas formas como amastigota, epimastigota e tripomastigota (CARVALHO GLB, et al., 2018).

A DC é uma infecção humana no qual tem como vetores os triatomíneos hematófagos, inseto também chamado de "barbeiro". Apresenta-se clinicamente por duas fases: a fase aguda, geralmente assintomática dificultando o diagnóstico, mas pode apresentar cefaléia, mal-estar, edema de face ou sinal de romanã, febre e hepatoesplenomegalia, e a fase crônica, podendo ter complicações cardíacas como cardiomegalia provocando insuficiência cardíaca, e digestivas como megacólon e megaesôfago que podem levar a morte (FERNANDES ALB, et al., 2018; VARGAS A, et al., 2016; MIZOGUTI IL, et al., 2018).

A miocardiopatia dilatada é uma das complicações mais temidas da DC, pois modifica a ejeção do ventrículo e altera o ritmo sinusal cardíaco. Diante desta patologia, o tratamento padrão ouro para os pacientes que evoluírem para insuficiência cardíaca é o transplante de coração, em que é feita uma estratificação de risco pela New York Heart Association (NYHA). Porém existem indivíduos que não podem realizar o transplante, como aqueles com hipertensão pulmonar, incompatibilidade $A B O$, patologia psiquiátrica grave, entre outros (RODRIGUES BA, et al., 2020).

A transmissão pode ocorrer pelo "barbeiro", transmissão vetorial, através de suas fezes em contato com mucosas ou ferimentos; por transmissão transfusional em que o agente etiológico pode persistir de forma funcional em temperaturas ambientes por 250 dias e a $4^{\circ} \mathrm{C}$ por 18 dias; através dos transplantes de órgãos; as formas congênitas que possui difícil diagnóstico precoce por ser assintomática e ocorre quando existe ninhos de amastigotas na placenta; acidentes laboratoriais; e a ingestão de alimentos contaminados como açaí, cana-de-açúcar, carne e sangue de animais infectados, sendo a mais frequente no Brasil com predomínio da região amazônica (VARGAS A, et al., 2016; SIMIONI PU, et al., 2019).

$\mathrm{Na}$ fase aguda da DCA o diagnóstico é realizado por meio da identificação do parasita por meio da observação do sangue a fresco, esfregaço ou gota espessa do paciente infectado. Por outro lado, na fase crônica o diagnóstico é baseado em exames sorológicos como ELISA, Hemaglutinação e a Imunofluorescência Indireta. $O$ tratamento da DC constitui o uso de antiparasitários como o benznidazol, de primeira escolha, e nifurtimox (CORREIA JR, et al., 2021). 
Para prevenir as formas de transmissão dos triatomíneos é importante evitar que o inseto se multiplique dentro de casa, e isso pode ser realizado pelo simples fato de colocar mosquiteiros e telas metálicas em janelas e portas para evitar a entrada do vetor, o uso roupas compridas com mangas e repelentes, principalmente à noite, por ser o período de maior infecção (SIMIONI PU, et al., 2019).

No Brasil a DC está presente em todo país, mas é predominante nas regiões norte e nordeste, pois são os locais onde encontram-se a polpa do açaí considerada umas das principais fontes de contaminação pelo Trypanossoma, havendo alto risco de armazenar o protozoário e ter a transmissão por via oral, em virtude da precariedade das condições sanitárias encontradas no local de produção. Além do açaí temos: cana-deaçúcar, pêssego, banana e batata (ANDRADE JKS, et al., 2020).

Foi possível perceber que os casos confirmados na região Norte do Brasil relacionado à DCA surgiram em forma de surtos. Esses surtos ocorreram de modo sazonal e aconteceram no mesmo período que a safra do açaí, geralmente no mês de agosto a novembro. Isso justifica a relação direta entre a infecção chagásica e o açaí, o alimento quando contaminado por fezes de animais hospedeiros e manipulado incorretamente aumenta a capacidade de transmissão, pois o protozoário $T$. cruzi é habilitado a resistir e sobreviver períodos de incubação e tratamentos térmicos e ainda assim manter a qualidade em multiplicar-se (MIZOGUTI IL, et al., 2018).

De acordo com o estudo de Júnior ASS, et al. (2017) demonstraram que os indivíduos de baixa escolaridade, pessoas que vivem em situação de vulnerabilidade social e econômica tendem a morar em periferias expostas a diversos fatores de risco como a falta de educação em saúde e infraestrutura sanitária que facilitam serem propícios a adquirir tal infecção. Porém, de acordo com Mizoguti IL, et al. (2018), desde os anos 2000 estão sendo realizadas políticas públicas, publicações de leis e documentos pelo Ministério da Saúde e da Agricultura com o intuito de regulamentar a qualidade da polpa do açaí para obter um controle higiênico e sanitário podendo assim prevenir a ocorrência de casos novos.

Em consonância ao exposto acima, Raiol IF, et al. (2019) observaram que houve um destaque no número de casos de pacientes com baixa escolaridade, o qual são pessoas mais vulneráveis socialmente, havendo uma maior dificuldade em divulgar as formas de transmissão do parasita para que esse entendimento proporcione a prevenção de tais infecções. Além disso foi notado que o açaí é a principal fonte de alimentação de população ribeirinha ou de baixa renda estabelecendo um maior risco de infecção da DCA.

É uma doença parasitária negligenciada sendo resultada das intervenções do homem ao meio ambiente, fazendo com que haja a destruição do habitat natural dos triatomíneos resultando no deslocamento desses insetos para habitarem-se em casas de pau a pique (CARVALHO GLB, et al., 2018). Atualmente, a DC se encontra entre as 17 doenças tropicais mais negligenciadas, refletindo a baixa prevenção contra o patógeno responsável pelo principal meio de transmissão da doença (CORREIA JR, et al., 2021).

O presente trabalho foi realizado com o objetivo de obter conhecimento e traçar o perfil epidemiológico através das variáveis como sexo, faixa etária, raça/cor, ano de maior prevalência, zona de residência, capital de notificação da DCA e casos confirmados segundo local provável infecção na região norte do Brasil no ano de 2015 a 2019.

\section{MÉTODOS}

Trata-se de um estudo epidemiológico retrospectivo exploratório de caráter descritivo, realizado através dos dados secundários do Sistema de Informação de Agravos de Notificação (SINAN) disponibilizados no sitio eletrônico do Departamento de Informática do Sistema Único de Saúde (DATASUS), onde foram estudadas as notificações de DCA na região Norte, no período de 2015 a 2019. O SINAN é um sistema que coleta e fornece dados gerados pelo Sistema de Vigilância Epidemiológica brasileiro sobre notificação e investigação de doenças e agravos da lista nacional de doenças de notificação compulsória. O DATASUS permite análises da situação sanitária contribuindo para tomadas de decisão baseadas em evidências e subsidiou manutenção de programas de saúde.

As variáveis estudadas foram: faixa etária, raça, sexo, zona de residência, capital, local provável infecção e ano de maior prevalência. As informações foram coletadas no dia 30 de janeiro de 2021 . Foram coletadas 
as estimativas de população residente na plataforma do DATASUS, sendo os dados calculados pelo Instituto Brasileiro de Geografia e Estatística (IBGE) e o Sistema de Informações sobre Mortalidade (SIM). A taxa de incidência (I) foi calculada a partir do número de casos novos notificados da DCA dividido pela população residente no local e ano, em seguida multiplicado 100 mil habitantes.

$$
I=\frac{N \text { úmero de casos }}{\text { População residente }} \times 100.000
$$

A taxa de prevalência $(P)$ da DCA na população em determinado período foi obtida multiplicando a quantidade de casos da variável em análise $(Y)$ por cem, em seguida dividindo o resultado pelo número total de casos.

$$
P=\frac{(Y \times 100)}{\text { Número total de casos }}
$$

O estudo adotou análise estatística descritiva, utilizando como ferramenta o Software Microsoft Excel® (2016). Para a análise e comparação das informações coletadas foi realizado o levantamento de 23 referências bibliográficas dos últimos cinco anos através de leitura de resumos, títulos e posteriormente leitura completa, publicados em jornais e revistas a partir dos critérios de seleção, que são: objetivo do estudo, ano de publicação e plataforma de publicação. Por se tratar de dados disponíveis em banco de domínio público, o presente estudo não necessitou da análise do Comitê de Ética.

\section{RESULTADOS}

No período compreendido entre os anos de 2015 e 2019 foram notificados 1744 casos de pacientes com DCA no Brasil, sendo a região Norte com maiores notificações do país. Observou- se a distribuição desses casos pelo país no qual a região Norte apresenta uma taxa de incidência de 8,84/100.000 ha, seguida da região Nordeste com incidência de $0,14 / 100.000$ ha, região Centro-Oeste com 0,03/100.000 ha, região Sudeste com 0,003/100.000 há, e na região Sul não há casos notificados sobre a patologia (Gráfico 1).

Gráfico 1 - Taxa de incidência de casos confirmados de Doenças de Chagas Aguda notificados no País no período de 2015 a 2019, segundo a região de notificação.

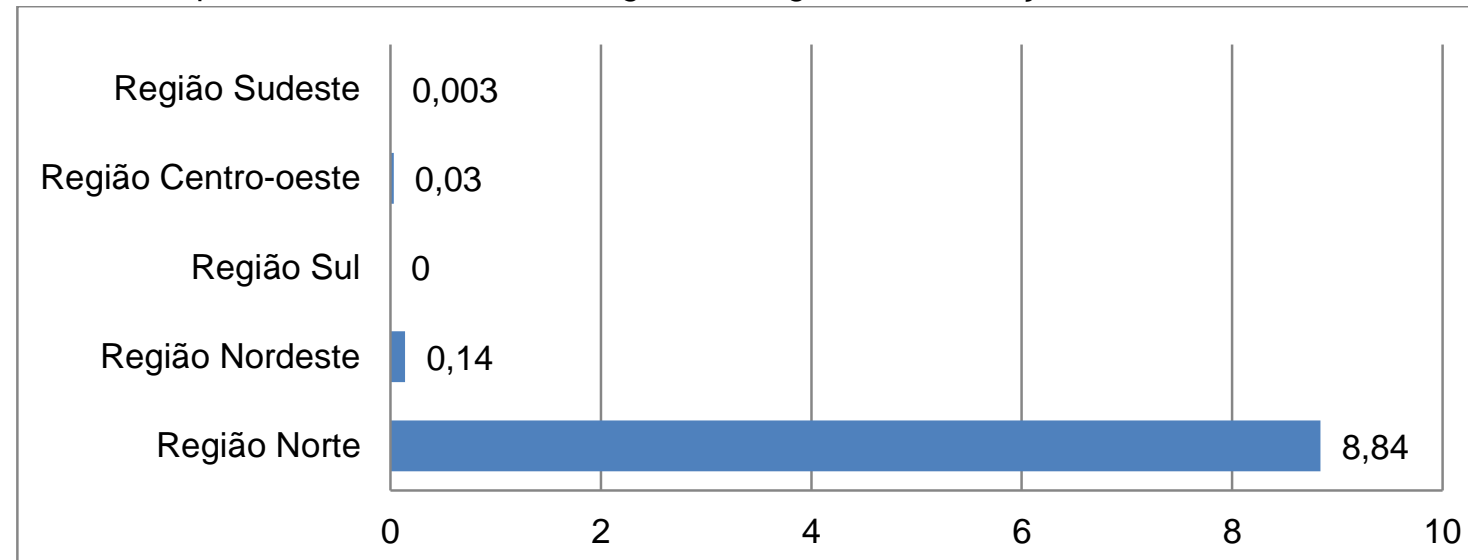

- Incidência de casos notificados de Doença de Chagas Aguda no Brasil entre os anos de 2015 e 2019 por 100.000 há

Fonte: Souza SB, et al., 2021; dados extraídos do Sistema de Informação de Agravos de Notificação (SINAN), 2020.

Notou-se que 2015 foi o ano de menor notificação apresentando 269 casos com uma incidência de 1,44/100.000 ha, já no ano de 2018 houve maior notificação de DCA com 354 casos notificados e uma incidência de 1,89/100.000 há (Gráfico 2). Observou-se a predominância das notificações de casos confirmados de DCA em indivíduos do sexo masculino com 897 notificações $(54,29 \%)$, sobre o sexo feminino com 755 notificações dentro do período da pesquisa (46,10\%) (Tabela 1). 
Gráfico 2 - Taxa de incidência de casos confirmados de Doenças de Chagas Aguda notificados na região norte do país entre 2015 a 2019, segundo a ano de notificação.

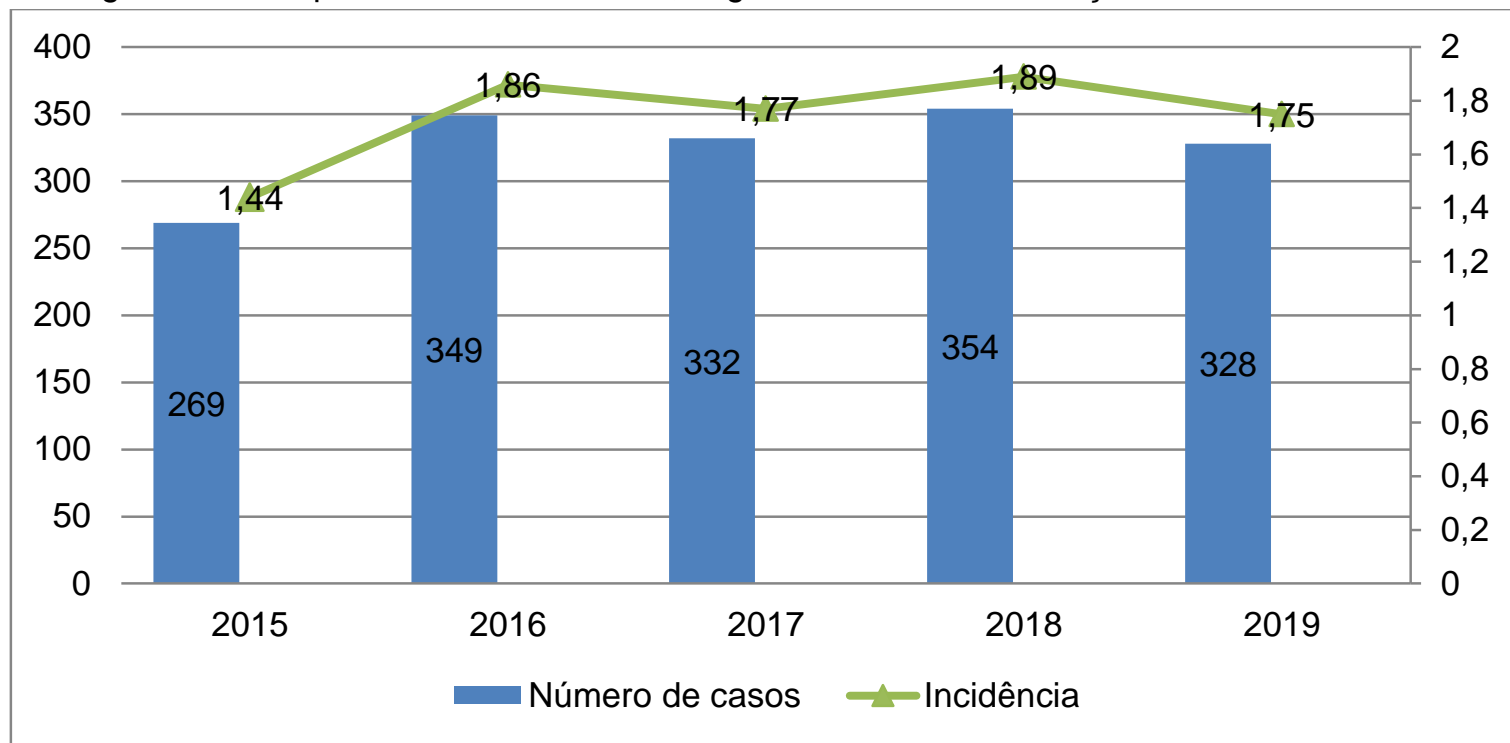

Fonte: Souza SB, et al., 2021; dados extraídos Sistema de Informação de Agravos de Notificação (SINAN), 2020.

No que concerne à variável raça/cor, constatou-se que a raça parda foi a que teve maior prevalência com 1399 casos $(84,78 \%)$, em seguida tem-se a raça branca com 128 casos $(7,74 \%)$, raça preta com 69 casos $(4,17 \%)$, indígenas com 10 casos $(0,60 \%)$ e a amarela com 4 casos ( $0,24 \%)$. Deste total de 1652,42 pessoas não declaram sua raça/cor. Em relação a faixa etária, observou-se maior concentração entre os indivíduos de 20-39 anos com 569 casos registrados (34,44\%) seguido dos indivíduos de 40-59 anos com 391 casos registrados $(23,66 \%)$ (Tabela 1$)$.

Tabela 1 - Taxa de prevalência de casos confirmados de Doenças de chagas Aguda notificadas segundo a variável sexo, raça/cor e faixa etária.

\begin{tabular}{cc}
\hline Variáveis & Porcentagem de acordo com a variável \\
\hline Sexo & $54,29 \%$ \\
\hline Masculino & $46,10 \%$ \\
\hline Feminino & \\
\hline Raça/Cor & $84,68 \%$ \\
\hline Parda & $7,74 \%$ \\
Branco & $4,17 \%$ \\
Preto & $2,54 \%$ \\
Ignorado & $0,60 \%$ \\
Indígena & $0,24 \%$ \\
Amarelo & \\
\hline Faixa etária & $1,27 \%$ \\
\hline 1 ano & $4,66 \%$ \\
$1-4$ anos & $7,56 \%$ \\
$5-9$ anos & $9,07 \%$ \\
$10-14$ anos & $9,07 \%$ \\
$15-19$ anos & $34,44 \%$ \\
$20-39$ anos & $23,66 \%$ \\
$40-59$ anos & $3,81 \%$ \\
$60-64$ anos & $2,54 \%$ \\
$65-69$ anos & $3,02 \%$ \\
$70-79$ anos & $0,84 \%$ \\
\hline 80 anos & \\
\hline SB, & at $2021 ;$ dados
\end{tabular}

Fonte: Souza SB, et al., 2021; dados extraídos do Sistema de Informação de Agravos de Notificação (SINAN), 2020. 
O maior número de casos de DCA relacionado à variável zona de residência tem destaque em pacientes que vivem na zona rural da região norte, com $51,39 \%$ dos casos, e zona urbana com apenas $46,67 \%$ dos casos (Gráfico 3).

Gráfico 3 - Taxa de prevalência de casos confirmados de Doenças de Chagas Aguda notificados segundo a zona de residência dos pacientes.

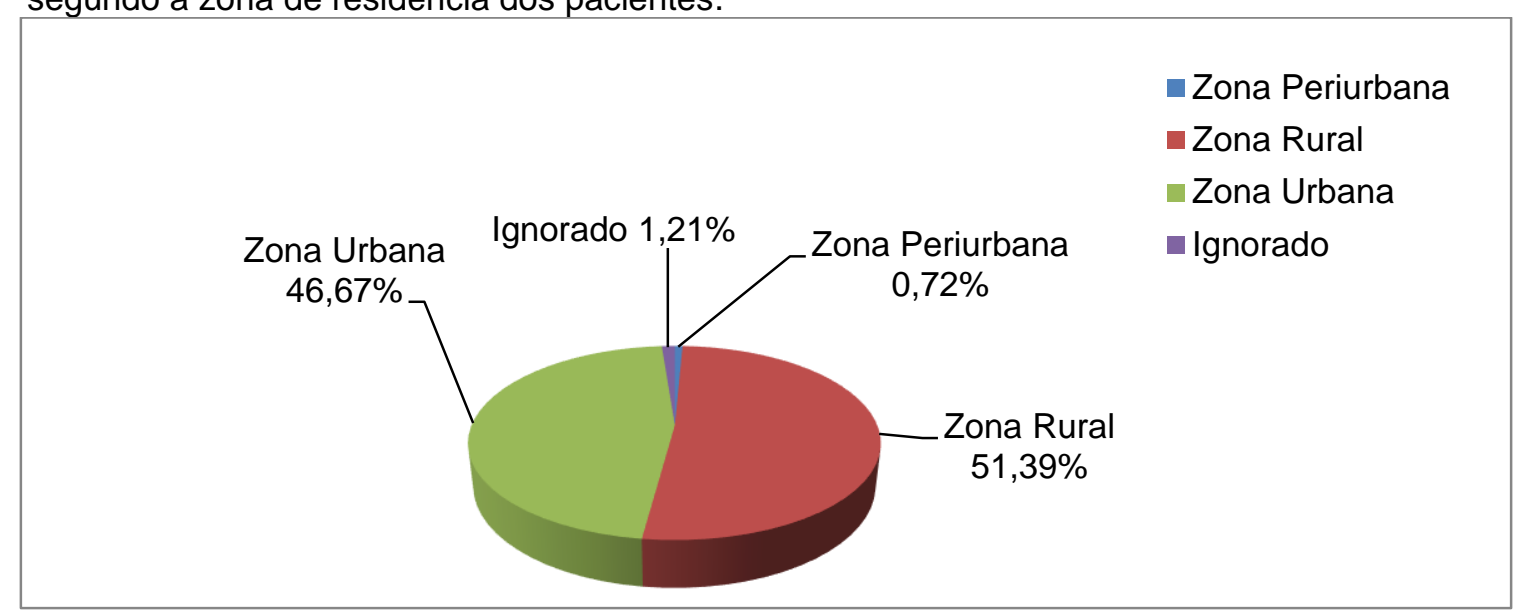

Fonte: Souza SB, et al., 2021; dados extraídos do Sistema de Informação de Agravos de Notificação (SINAN), 2020.

O Gráfico 4 demonstrou que a capital Belém possui o maior número de casos entre as capitais da região Norte do país, com 105 notificações. Porém, a capital Macapá possui uma maior incidência com uma taxa de 14,42/100.000 ha em comparação a Belém que possui apenas 7,00/100.000 ha.

Gráfico 4 - Taxa de incidência de casos confirmados de Doenças de Chagas Aguda notificados segundo a capital de notificação dos pacientes.

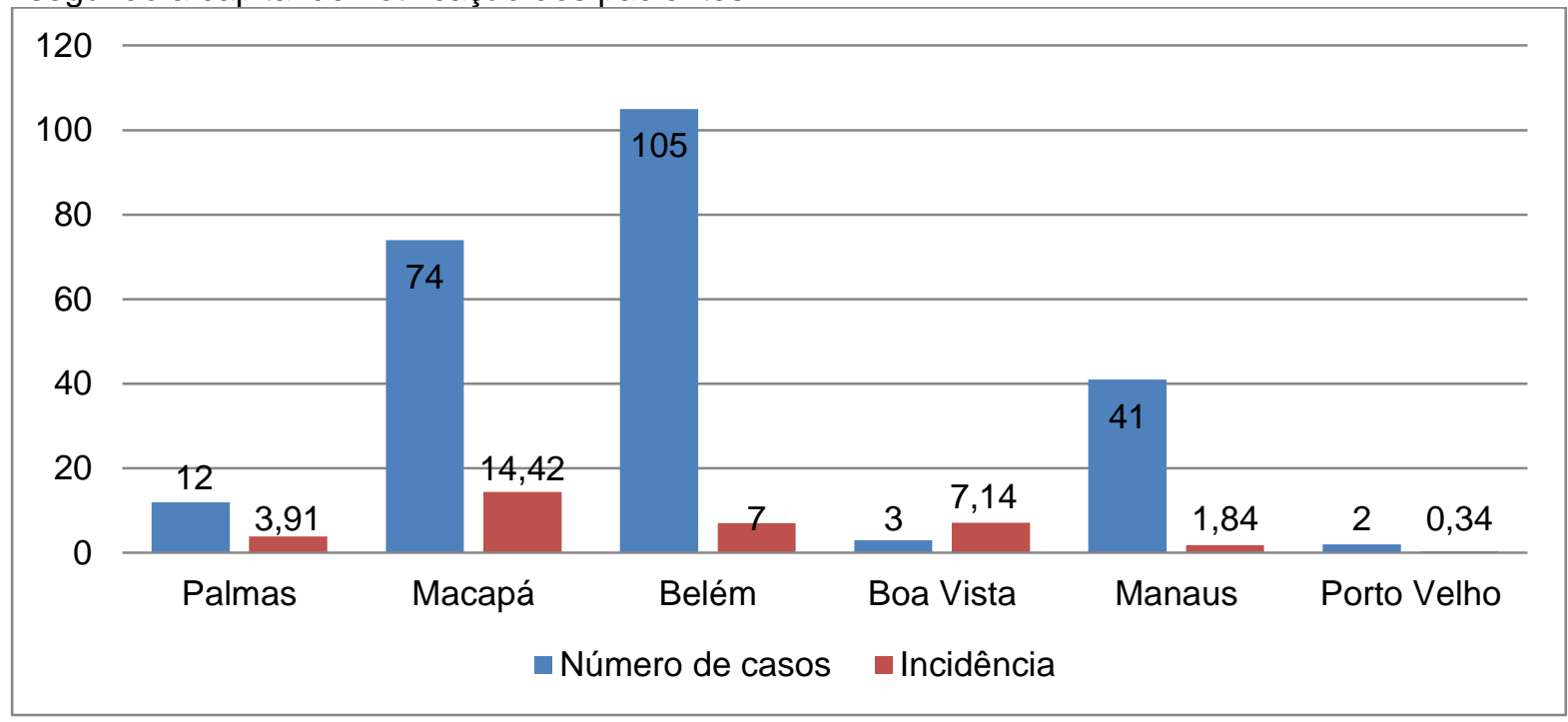

Fonte: Souza SB, et al., 2021; dados extraídos do Sistema de Informação de Agravos de Notificação (SINAN), 2020.

O Gráfico 5 demonstra a taxa de incidência dos casos confirmados de acordo com o provável local de infecção podendo ser ele domiciliar, unidade de hemoterapias, outros e os ignorados/ brancos, aqueles que não tiveram informações. Notou-se um número de casos elevados com provável infecção no local de domicílio com 953 casos e uma incidência de 5,10/100.000 ha. Já o local de unidade de hemoterapia obteve somente 6 casos no período de 2015 a 2019 com uma incidência de 0,03/100.000 ha, os outros locais houve um número de 101 confirmações e incidência de 0,54/100.000 ha, e do total de 1652 casos 592 não foram determinados o local de infecção. 
Gráfico 5 - Taxa de incidência de casos confirmados de Doenças de Chagas Aguda notificados segundo local provável de infecção.

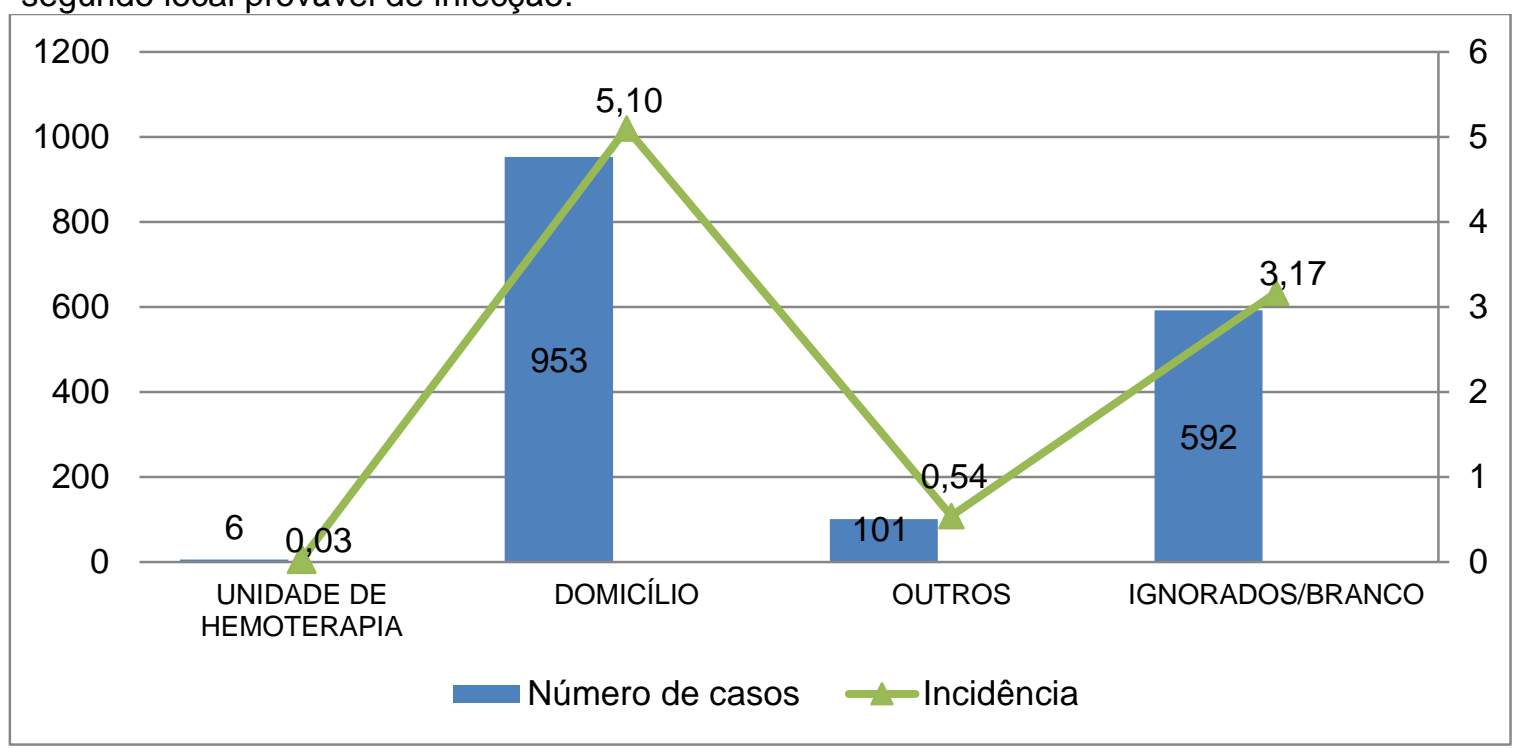

Fonte: Souza SB, et al., 2021; dados extraídos do Sistema de Informação de Agravos de Notificação (SINAN), 2020.

\section{DISCUSSÃO}

Identificou-se, a partir dos dados coletados no DATASUS, que a região Norte possui a maior taxa de incidência de morbidade relacionada à DCA, quando se comparado com as outras regiões brasileiras. Isso já foi observado em outros estudos, como o de Dias JCP, et al. (2016) que encontraram uma prevalência de $91,1 \%$ dos casos na região Norte e o estudo realizado por Felix EBG, et al. (2020) que obtiveram uma proporção de $92,1 \%$ de casos.

De acordo com Souza DSM, et al. (2016) essa incidência de casos de DCA na região Norte justificou-se pela contaminação por meio do suco do açaí, comida tradicional e mais prevalente na região, com maior incidência nos meses de setembro e outubro, período no qual ocorre a sua colheita. A contaminação ocorreu devido a presença dos triatomíneos, inseto vetor da DC, próximo às palmeiras associado à manipulação inadequada da fruta. Em concordância Mizogutl IL, et al. (2018) acrescentaram que o protozoário possui capacidade de sobreviver na polpa da fruta mesmo submetido a diversos tratamentos térmicos, por diferentes períodos de incubação, mantendo sua potencialidade de se multiplicar e provocar a doença. Além disso, observaram que o tratamento prévio do açaí é um fator protetivo, visto que indivíduos que consumiram açaí refrigerado não desenvolveram a patologia.

Em relação ao período de pesquisa notou-se que no ano entre 2015 a 2019 houve uma oscilação na taxa de incidência durante os anos. Em seguida, comparando o ano de 2015 e de 2019 constatou-se um aumento de 21,93\%, nos números de casos novos. Entretanto, o ano de 2018 foi o que teve maior incidência e quando se comparado com o ano de 2015 percebeu-se um aumento de 31,59\%. Desse modo, evidenciou que ao longo dos cinco anos determinados para a pesquisa, a taxa de incidência de DC apresentou-se oscilante na região Norte, o que pode ser justificado pela falta de controle dos fatores de risco da doença.

A prevalência da doença foi maior no sexo masculino sendo um fato que coincide com outros estudos, por exemplo, o realizado por Gomes G, et al. (2020) que obtiveram em seu estudo $54,78 \%$ de casos notificados entre o sexo masculino, o estudo de Cardoso LP, et al. (2020), que revelaram uma incidência entre homens com 154,96/100 mil habitantes enquanto as mulheres teve 133,87/100 mil habitantes casos novos, e o estudo de Santos FAC, et al. (2020) em que afirmaram a predominância no sexo masculino com uma diferença de 100 casos em relação ao feminino.

Esses dados se devem a uma maior exposição e contato em áreas endêmicas do vetor por indivíduos do sexo masculino devido a atividades laborais realizadas e locais de moradia que aumentam o risco de 
transmissão do Trypanosoma cruzi. A agricultura como profissão se tornou um fator de risco comum, sendo executada principalmente por indivíduos do sexo masculino, o que contribui para a explicação dos dados apresentados (CARDOZO EJS, et al., 2017; CARDOSO LP, et al., 2020).

Nessa pesquisa epidemiológica verificou-se que a raça parda representa o maior número de casos confirmados na região Norte, da mesma maneira que está presente nos estudos de Felix EBG, et al. (2020) e Cardoso LP, et al. (2020), que alcançaram uma predominância de $82,3 \%$ e $82,76 \%$ dos casos sendo cor/ raça parda, respectivamente, justificando esses dados por estarem interligados com a variável socioeconômica.

Entende-se que há uma grande variabilidade no termo parda quanto a sua origem étnica no Brasil, a qual influencia de forma significativa nos estudos epidemiológicos, sendo assim diversos fatores socioeconômicos são considerados como: nível de instrução, nível de renda, hábitos e aspectos fenotípicos. Essa avaliação permite identificar grupos desfavorecidos devido iniquidades sociais e desigualdades na saúde no Brasil, que são frequentemente observadas nesse grupo racial e que influencia diretamente na prevalência de casos entre os indivíduos que se determinam pardos na região norte do país (ALENCAR MMF, et al., 2020).

Dentre os indivíduos acometidos pela doença, notou-se que a faixa etária mais acometida foi entre $20 \mathrm{e}$ 39 anos, seguida pela faixa etária de 40 e 59 anos, demostrando que quanto maior a faixa etária maior o risco de contaminação, devido ao maior tempo de exposição ao patógeno. Ao comparar com outras bibliografias houve compatibilidade com o estudo realizado por Santos VRC, et al. (2018) que obtiveram como resultado a prevalência de $69 \%$ dos casos entre a idade de 18 e 60 anos, e Gomes G, et al. (2020) que ao analisarem 1515 casos evidenciaram 521 casos em pacientes com 20 a 39 anos, seguida por 369 casos em indivíduos de 40 a 59 anos de idade.

Ademais, foi evidenciado no Boletim Epidemiológico de Doença de Chagas, publicado pela Secretaria de Saúde do Estado da Bahia, maior prevalência de casos notificados na faixa etária de 20 a 39 anos, apresentando 53,4\% das notificações, seguida por 39,4\% em indivíduos entre 40 e 59 anos (BRASIL, 2019).

Correia JR, et al. (2021) perceberam uma concordância com o estudo, demonstraram que os casos de DCA acometiam principalmente pacientes entre 19 a 59 anos, com disseminação por via oral, indivíduos com baixa escolaridade do sexo masculino, justificado por trabalharem em contato com animais e lavouras e estando suscetíveis a contaminação.

No presente estudo, ao analisar a zona de residência de maior prevalência verificou-se maior porcentagem para zona rural $(51,39 \%)$, que corrobora com a pesquisa realizada por Pereira CML, et al. (2017) que ao investigar o estado do Pará, registraram a prevalência de $74,1 \%$ de contaminação na zona rural em comparação com a zona urbana. Além disso, Cardoso LP, et al. (2020) ao realizarem uma pesquisa epidemiológica no município de Abaetetuba, estado do Pará, registraram maior incidência na zona rural, apresentando uma taxa de 168,67/100hab, fortalecendo o resultado da presente pesquisa.

A partir das incidências calculadas, segundo a variável capital de notificação, notou-se maior prevalência de casos na capital Belém, no entanto, maior incidência na capital do estado de Amapá. Em ambas as situações, a transmissão oral pela ingestão do caldo de cana ou suco de açaí contaminado, principalmente em populações distantes das regiões metropolitanas e ribeirinhas, justifica as informações de prevalência e incidência anteriormente citadas (PARENTE MF, et al., 2020; CARDOSO LP, et al., 2020). Desse modo, a contradição dos dados de prevalência e incidência, entre Belém e Macapá, supostamente relaciona-se a uma maior vigilância sanitária na capital do estado do Pará, pois ambos compartilham os mesmos fatores de risco e meios de transmissão.

A DC apresenta uma evolução otimista quando realizado o diagnóstico e tratamento precoce, sendo o conhecimento dos fatores de riscos de extrema importância para estabelecer métodos de prevenção. É necessário pontuar que idosos que apresentam a doença e possuem comorbidades estão sujeitos a evoluírem para um pior quadro clínico, especialmente aqueles que possuem doenças crônicas cardiovasculares e gastrointestinais, considerando que são os sistemas de maiores complicações da DC (JÚNIOR ASS, et al., 2017). 


\section{CONCLUSÃO}

Foi evidenciado o perfil epidemiológico dos pacientes infectados pelo Trypanossoma cruzi, com maior predominância no sexo masculino, indivíduos que se denominam raça parda, na faixa etária de 20 a 39 anos, seguido por indivíduos de 40 a 59 anos, predominantes na zona rural. Ademais, identificou-se que a capital Macapá possui maior incidência de casos entre as capitais da região pesquisada. Desse modo, esta pesquisa possibilitou pontuar fatores importantes para detecção de surtos e epidemia, contribuindo para a vigilância de saúde, ações de combate e medidas de prevenção. Por fim, espera-se que a pesquisa venha ajudar futuros estudos relacionados ao Trypanossoma cruzi com a coleta de dados recentes.

\section{REFERÊNCIAS}

1. ALENCAR MMF, et al. Epidemiologia da Doença de Chagas aguda no Brasil de 2007 a 2018 . Research, Society and Development, 2020; 9(10): e8449109120-e8449109120.

2. ANDRADE JKS, et al. Qualidade microbiológica de polpas de açaí comercializadas em um estado do nordeste brasileiro. Revista Brasileira de Higiene e Sanidade Animal,2020; 14(2): 226-236.

3. BRASIL. Boletim epidemiológico de Doença de Chagas. 2019. Disponível em: http://www.saude.ba.gov.br/wpcontent/uploads/2017/11/2019-Boletim-epidemiol\%C3\%B3gico-Doen\%C3\%A7as-de-Chagas-n.-01-3.pdf. Acessado em: 26 de janeiro de 2020.

4. BRASIL, Ministério da Saúde. Banco de dados do Sistema Único de Saúde-DATASUS. Disponível em: http://tabnet.datasus.gov.br/cgi/deftohtm.exe?sinannet/cnv/chagasbr.def. Acesso em: 20. Jan. 2021.

5. CARDOSO LP, et al. Spatial distribution of Chagas disease and its correlation with health services. Revista da Escola de Enfermagem da USP, 2020; 54: e03565.

6. CARDOZO EJS, et al. Perfil epidemiológico dos portadores de doença de chagas: dos indicadores de risco ao processo de enfrentamento da doença. Arquivos de Ciências da Saúde, 2017; 24(1): 41 -46.

7. CARVALHO GLB, et al. Doença e Chagas: Sua transmissão através do consumo de açaí. Acta de Ciências e Saúde, 2018; 1(1): 1-13.

8. CORREIA JR, et al. Doença de Chagas: aspectos clínicos, epidemiológicos e fisiopatológicos. Revista Eletrônica Acervo Saúde, 2021; 13(3): 1-7.

9. DIAS JCP, et al. II Consenso Brasileiro em doença de Chagas, 2015. Epidemiologia e Serviços de Saúde, 2016; 25:786.

10. FELIX EBG, et al. Doença de chagas no Brasil: estudo transversal com base nos dados referentes ao período de 2017-2018. Revista Interfaces: Saúde, Humanas e Tecnologia, 2020; 8(2): 561-570.

11. FERNANDES ALB, et al. Incidência e prevalência da doença de chagas no Brasil. CIPEEX, 2018; 2:978-983.

12. GOMES G, et al. Perfil epidemiológico da Doença de Chagas aguda no Pará entre 2010 e 2017. Pará Research Medical Journal, 2020; 4: e29.

13. JÚNIOR ASS, et al. Análise espaço-temporal da doença de Chagas e seus fatores de risco ambientais e demográficos no município de Barcarena, Pará, Brasil. Revista Brasileira de epidemiologia, 2017; 20: 742-755.

14. MIZOGUTI IL, et al Doença de Chagas: A culpa é do Açaí. Boletim informativo da Universidade de São Paulo, 2018; 1(1): 1-10.

15. PARENTE MF, et al. Cenário epidemiológico da Doença de Chagas no Estado do Pará, Brasil. Brazilian Journal of Health Review, 2020; 3(1): 1223-1234.

16. PEREIRA CML, et al. Perfil clínico e epidemiológico da doença de chagas aguda no estado de Minas Gerais. Revista de Atenção à Saúde, 2017; 15(52): 49-54.

17. RAIOL IF, et al. Inovações tecnológicas como instrumento de educação para a higienização do açaí em uma comunidade ribeirinha da Amazônia: um relato de experiência. Revista Eletrônica Acervo Saúde, 2019; 27 : e920.

18. RODRIGUES BA, et al. Reativação da doença de Chagas pós-transplante cardíaco. Revista Eletrônica Acervo Científico, 2020; 12: e4652.

19. SANTOS FAC, et al. Avaliação da prevalência e do perfil epidemiológico da doença de chagas aguda entre 2014 e 2017 no estado do Pará, Brasil. Brazilian Journal of Health Review, 2020; 3(4): 8974-8982.

20. SANTOS VRC, et al. Acute Chagas disease in the state of Pará, Amazon Region: is it increasing?. Memórias do Instituto Oswaldo Cruz, 2018; 113(5): e170298.

21. SIMIONI PU, et al. Métodos de Prevenção e Tratamento para a Doença de Chagas. Ciência \& Inovação, 2019; 4(1): 61-67.

22. SOUZA DSM, et al. Aspectos Anatomopatológicos da Miocardite Chagásica Aguda por Transmissão Oral. Arquivos Brasileiros de Cardiologia, 2016; 107(1): 77-80.

23. VARGAS A, et al. Investigação de surto de doença de Chagas aguda na região extra-amazônica, Rio Grande do Norte, Brasil, 2016. Cadernos de saúde pública, 2018; 34: e00006517. 